\title{
Article
}

\section{Chemical characteristics of Amazonian Dark Earth in Santarem, Brazil}

Douglas Sousa da Silva', Julio Cesar Amaral Cardoso", Brainy César Castro Lima', Arthur Abinader Vasconcelos ${ }^{1}$, Lilian Rebellato ${ }^{2}$, Denise Pahl Schaan ${ }^{3}$, Denise Maria Cavalcante Gomes ${ }^{4}$, Rafael da

Rosa Couto ${ }^{5}$, Gustavo Brunetto ${ }^{6}$, Paulo Sérgio Taube ${ }^{1 *}$

${ }^{1}$ Institute of Biodiversity and Forests, Federal University of Western Pará, Santarém, PA, CEP 68035-110, Brazil

${ }^{2}$ Institute of Social Science, Federal University of Western Pará, Santarém, PA, CEP 68040-070, Brazil

${ }^{3}$ Institute of Philosophy and Human Sciences, Federal University of Pará, Belém, PA, CEP 66075-110, Brazil

${ }^{4}$ Department of Anthropology, Federal University of Rio de Janeiro, Rio de Janeiro, RJ, CEP 20940-040, Brazil

${ }^{5}$ Nucleus of Education, Research and Extension in Agroecology, Federal University of

Santa Catarina, Florianópolis, SC, CEP 88034-000, Brazil

${ }^{6}$ Department of Soil Science, Federal University of Santa Maria, Santa Maria, RS, CEP 97105-900, Brazil

\section{Graphical Abstract}

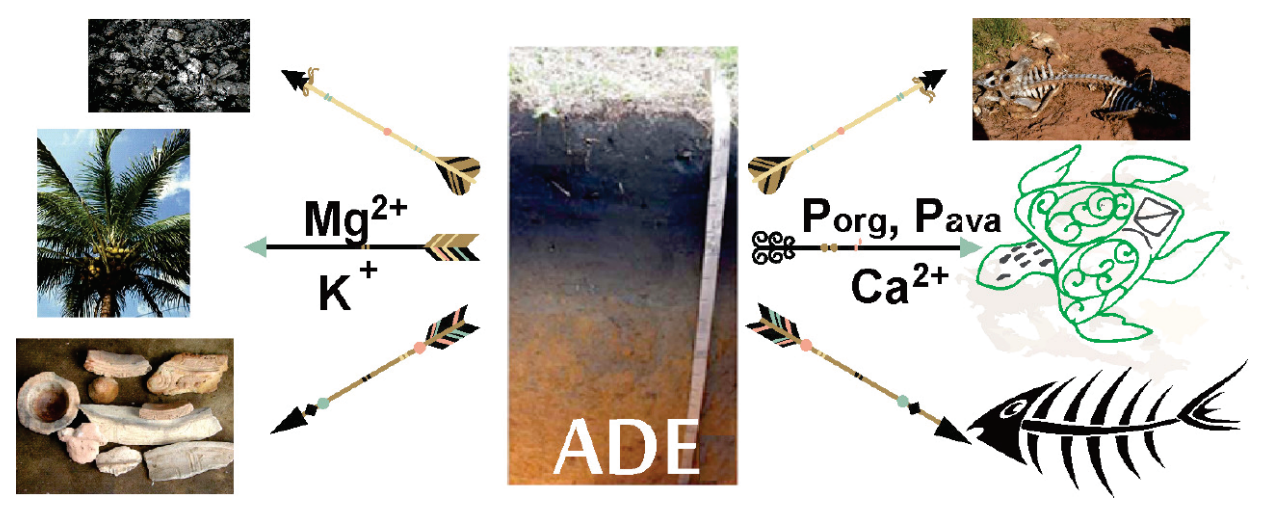

Possible sources of incorporation of $\mathrm{P}, \mathrm{Ca}, \mathrm{Mg}$ and $\mathrm{K}$ into the Amazonian Dark Earth The Indian-style arrows represent the incorporation in the pre-colonial period. The main sources of $\mathrm{P}$ and $\mathrm{Ca}$ (carbonate and phosphate) are bones of fish and other animals, and carapaces of chelonians.

This research aimed at to compare some chemical characteristics $\left(\mathrm{pH}\right.$ in water, and $\mathrm{KCl}, \mathrm{Ca}^{2+}, \mathrm{Mg}^{2+}, \mathrm{K}^{+}$, $\mathrm{Al}^{3+}$, organic phosphorus ' $\mathrm{P}_{\text {org }}$ and available phosphorus ' $\mathrm{P}_{\text {ava }}$ ') of anthropogenic and non-anthropogenic soils. Soil samples were collected at five depths: 0-20, 20-40, 40-60, 60-80 and 80-100 cm in two archaeological sites and one non-anthropogenic area. The analysis revealed that the anthropogenic soils presented higher amount of $\mathrm{P}_{\text {ava }}$ than the non-anthropogenic ones, and that these amounts presented a positive correlation with the $\mathrm{Ca}^{2+}$ contents, which reflects the incorporation of animal bones, mainly fish to these soils. However, the non-homogeneous distribution of these species and the low $\mathrm{K}^{+}$contents found in most samples may be limiting for some cultures. Finally, it was possible to differentiate samples of the anthropogenic soils from non-anthropogenic ones by using the values of $\mathrm{pH}$ in water and $\mathrm{KCl}$, as well as the $\mathrm{P}_{\text {org }}$ and $\mathrm{P}_{\text {ava }}$ contents.

Key words: phosphorus availability, phosphorus fractionation, macronutrients, soil pH. 


\section{Article}

\section{INTRODUCTION}

Most soils of the Amazonic region in Brazil, Bolivia, Colombia, Peru and Ecuador are acid, presenting low natural fertility [1-2]. In this same region, it is possible to find anthropogenic soils with plaggen epipedon horizon, normally associated with high fertility, thus characterizing the so called Amazonian Dark Earths (ADE) [3-4].

ADEs present higher amounts of calcium, manganese and phosphorus in relation to the nonanthropogenic Oxisols and Ultisols [4-6]. They also present high amounts of stable organic matter, being rich in pyrogenic carbon (black carbon) $[4,7]$ thus the presence of ceramics and/or lithic material is a striking feature [8-9]. Their high fertility is due to the incorporation of inorganic (bones and ashes) and organic (plants and animals) residues into the soil $[4,10]$, which results in soils with moderate acidity and low levels of exchangeable aluminum [11].

ADEs are generally found near riverbanks and have A horizons deeper in comparison with the nonanthropogenic adjacent soils [12]. They also are characterized by good drainage, high water availability, and low density, ideal conditions for aeration, porosity and hydraulic conductivity, which favor water penetration and gas exchange [13].

Unlike most non-anthropogenic soils, ADEs generally present high levels of available phosphorus $\left(P_{\text {ava }}\right)$, which is one of the most important characteristics for their agricultural use in the amazonic region [14]. The high amount of phosphorus reflects the incorporation of large quantities of fish bones, turtle shells, and other animals $[9,15]$. In spite of the high levels of $\mathrm{P}, \mathrm{Ca}$ and $\mathrm{Mg}$, several studies of chemical characterization of ADE soils have shown that $K$ is normally found in low concentrations in these soils, which may cause deficiency of this nutrient for certain crops [14,16-17].

Moreover, ADEs are relevant in the search for alternatives to sustainable agricultural practices in the Amazon [11,18]. In the region of Santarem, Brazil, there are several archeological sites, some with a large extension; however, most of the studies already undertaken in these areas emphasize mainly archeological questions. Thus, in order to generate information about the fertility of ADEs in urban areas and to understand the heterogeneity of these soils, the following hypothesis can be established: ADE soils present high levels of organic and available phosphorus, however, the non-homogeneity in the distribution of these species and the possible potassium deficiency may limit their use for some cultures. In this context, this study aimed to evaluate chemical attributes in soils from two archaeological sites containing ADEs and an adjacent non-anthropogenic soil.

\section{MATERIAL AND METHODS Location of study area}

The study areas were located in Santarem, a city in the western region of the state of Pará, Brazil (Figure 1). Soil samples were collected at two archaeological sites: 1) ADE 1 ( $2^{\circ} 25^{\prime} 06^{\prime \prime S}$ and 54 $\left.{ }^{\circ} 44^{\prime} 19^{\prime \prime} \mathrm{W}\right)$, which has a predominant vegetative cover of grasses (Paspalum sp.) and other species, such as: Spondias mombin, Psidium sp., Acrocomia aculeata, Euterpe edulis mart, Byrsonima basiloba, Astrocarium tucuma, Pouteria macrophylla [19] and large fruit trees, such as e.g. cashew and hose tree [20]; 2) ADE 2 ( $2^{\circ} 25^{\prime} 17^{\prime \prime} S$ and $54^{\circ} 44^{\prime} 03^{\prime \prime} \mathrm{W}$ ), which has a predominant vegetative cover of grasses (Paspalum sp.), species Mangifera indica, Syzygium malaccense, Anacardium occidentale and Cyperus sp and; 3) an adjacent area (nonanthropogenic), with a predominant vegetative cover of grasses (Echinochloa pyramidalis and Paspalum sp.), Mangifera indica, Spondias mombin and Anacardium occidentale trees (2 $25^{\prime} 39^{\prime \prime S}$ and 54 $\left.44^{\prime} 19^{\prime \prime} \mathrm{W}\right)$.

The climate of the region according to the Köppen classification is Ami (tropical humid), average annual temperature of $25.5{ }^{\circ} \mathrm{C}$; relative air humidity around $90 \%$ and annual average rainfall of $2318 \mathrm{~mm}$, with pronounced variations during the year [21]. Usually, higher volumes of precipitation are observed during the January and May period. 


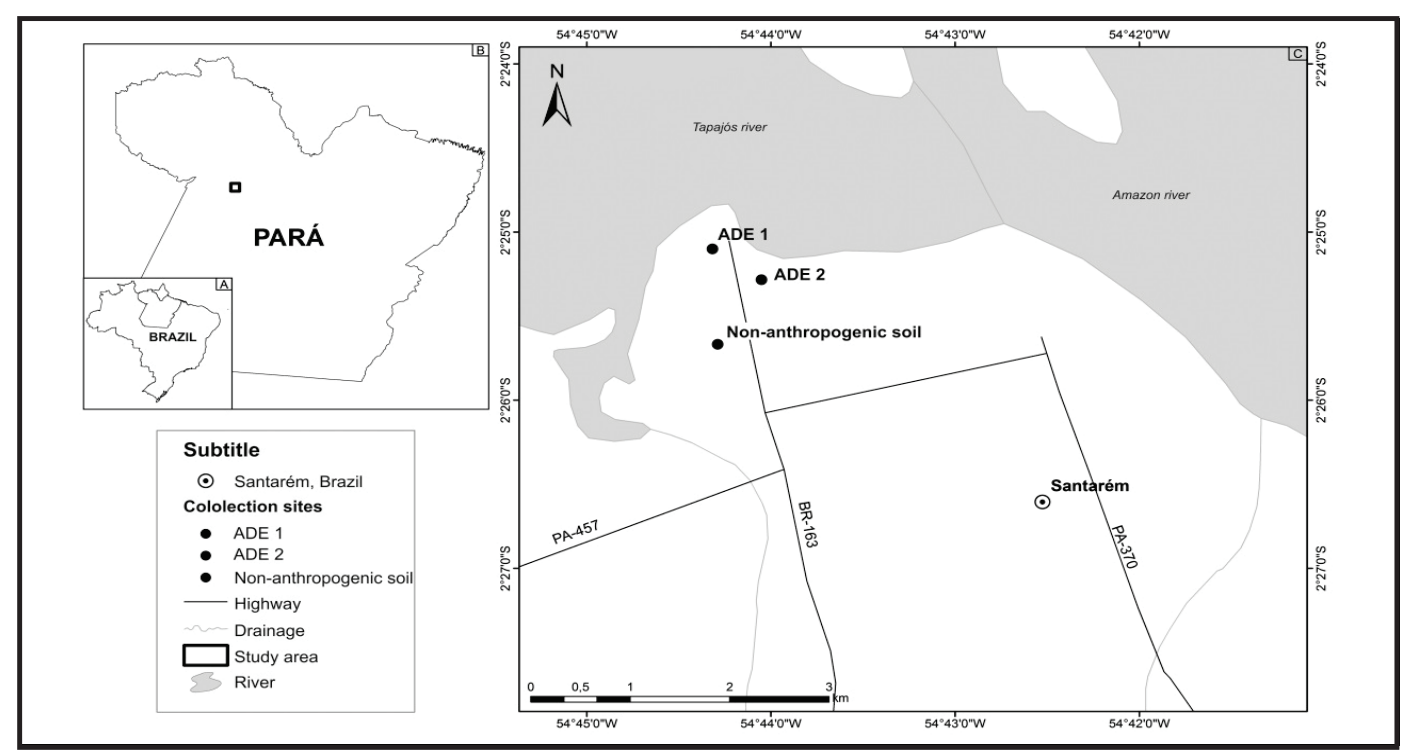

Figure 1. Geographical location of the areas evaluated

Santarem is located in the central portion of the Sedimentary Basin of Amazonas, with most of its territory formed in the upper section of the Cretaceous / Tertiary period (Alter do Chão Formation). It is characterized by ruffled and reddish sediments with predominance of fine and thick sandstones with frequent cross-stratification [22]. The area presents Lithographs of the Cretaceous/Tertiary and Quaternary Periods, being the first one represented by lithologies of the Alter do Chão formation, formed by fine and medium sandstones, siltstones and kaolinitic, yellow, red and white argillites. This formation presents tabular surfaces, thus a flat relief. The predominant soils in the region are: typical dystrophic yellow latosol, typical dystrophic argisol and gleysol [22].

\section{Soil collection and analysis}

In each studied area, a $30 \times 30$ m plot was demarcated. In each soil, samples were collected in five points at five depths: 0-20, 20-40, 40-60, 60-80 and 80-100 cm. All soil samples were dried in air, macerated and sifted ( $2 \mathrm{~mm}$ mesh) to obtain the fine earth fraction (FEF). The soil was then packed in polyethylene bags and reserved for further analysis.

Soil particle size analysis was carried out by using $20 \mathrm{~g}$ of soil and $10 \mathrm{~mL}$ of a $0.1 \mathrm{~mol} \mathrm{~L}^{-1} \mathrm{NaOH}$ solution [23]. The soil:extractor suspension was subjected to mechanical stirring at $240 \mathrm{rpm}$ during $15 \mathrm{~min}$. Thereafter, the clay and sand fractions (coarse and fine) were separated by sedimentation and sieving, respectively, and the silt fraction was calculated by difference between the total amount and sum of clay plus sand contribution.

$\mathrm{pH}$ values in water and $1.0 \mathrm{~mol} \mathrm{~L}^{-1} \mathrm{KCl}$ was potentiometrically measured, using a 1.0:2.5 soil:solution ratio [23]. The $\Delta \mathrm{pH}$ was calculated as the difference between the $\mathrm{pH}$ in $\mathrm{KCl}$ and $\mathrm{pH}$ in water.

The exchangeable cations $\left(\mathrm{Ca}^{2+}\right.$ and $\left.\mathrm{Mg}^{2+}\right)$ were extracted with a $1.0 \mathrm{~mol} \mathrm{~L}^{-1} \mathrm{KCl}$ aqueous solution, and the in soil:solution proportion was 1:20 (w/v) [24]. These cations were determined in a model 3110 atomic absorption spectrometer (Perkin Elmer, Texas, USA) equipped with a flame atomization. $\mathrm{K}^{+}$was extracted with aqueous solution Mehlich-1 $\left(0.05 \mathrm{~mol} \mathrm{~L}^{-1} \mathrm{HCl}\right.$ plus $\left.\mathrm{H}_{2} \mathrm{SO}_{4} 0.0125 \mathrm{~mol} \mathrm{~L}^{-1}\right)$ [24] and, quantified in a model DM61 Digimed flame photometer DM61 (Sao Paulo, SP, Brazil) equipped with an interference filter for determining $\mathrm{K}, \mathrm{Na}$, Li and $\mathrm{Ca}$.

$\mathrm{Al}^{3+}$ in the $\mathrm{KCl}$ extract was determined by titration with a $0.025 \mathrm{~mol} \mathrm{~L}^{-1} \mathrm{NaOH}$ solution [18]. Available phosphorus $\left(\mathrm{P}_{\text {ava }}\right)$ was extracted with aqueous solution Mehlich-1 in a 1:10 (soil:solution, w/v) proportion [24]. The organic phosphorus $\left(P_{\text {org }}\right)$ was extracted with $0.5 \mathrm{~mol} \mathrm{~L}^{-1} \mathrm{H}_{2} \mathrm{SO}_{4}$ solution according to Olsen and Sommers [25]. Both phosphorus fractions were quantified according Murphy and Riley [26]. 


\section{Article}

\section{Statistical analysis}

The analytical results were submitted to principal component analysis using the software MINITAB $14^{\odot}$ (Minitab, State College, PA, USA). The analysis of the effects between the areas was performed by considering the variability resulting from the values obtained in the samples evaluated in triplicate and expressed from the standard deviation of the mean.

\section{RESULTS AND DISCUSSION}

The analysis of soil texture showed the predominance of the sand fraction in all depths of the analyzed soils, with higher amounts in the ADE 1 (Table I). These results were similar those found by Lima et al. (2002), when analyzing soils with ADE horizon in the western Amazonia [15].

Table I. Particle size distribution of the analyzed soils

\begin{tabular}{|c|c|c|c|c|}
\hline \multirow{2}{*}{ Soil } & \multirow{2}{*}{ Depth } & \multicolumn{3}{|c|}{ Granulometry $\left(\mathrm{g} \mathrm{kg}^{-1}\right)$} \\
\hline & & Sand & Silt & Clay \\
\hline \multirow{5}{*}{ ADE 1} & $0-20$ & 835 & 87 & 78 \\
\hline & $20-40$ & 820 & 88 & 92 \\
\hline & $40-60$ & 857 & 65 & 78 \\
\hline & $60-80$ & 868 & 63 & 69 \\
\hline & $80-100$ & 867 & 43 & 90 \\
\hline \multirow{5}{*}{ ADE 2} & $0-20$ & 662 & 188 & 150 \\
\hline & $20-40$ & 656 & 139 & 205 \\
\hline & $40-60$ & 657 & 136 & 207 \\
\hline & $60-80$ & 678 & 119 & 203 \\
\hline & $80-100$ & 670 & 131 & 199 \\
\hline \multirow{5}{*}{$\begin{array}{l}\text { Non-anthropogenic } \\
\text { soil }\end{array}$} & $0-20$ & 770 & 88 & 142 \\
\hline & $20-40$ & 778 & 77 & 145 \\
\hline & $40-60$ & 766 & 85 & 149 \\
\hline & $60-80$ & 772 & 72 & 157 \\
\hline & $80-100$ & 783 & 77 & 140 \\
\hline
\end{tabular}

The $\mathrm{pH}$ values in water and $\mathrm{KCl}$ were not homogeneous intra and extra anthropogenic soils, i.e, $\mathrm{pH}$ oscillated with depth in the same ADE and varied from ADE to ADE (Tables II and III). The $\mathrm{pH}$ values in water ranged from 5.04 to 8.22 (Table II) and 4.56 to 6.12 (Table III), whereas those in $\mathrm{KCl}$ ranged from 4.62 and 6.70 (Table II) and 3.53 and 5.38 (Table III), in ADE 1 and ADE 2, respectively. On the other hand, in the non-anthropogenic soil, the $\mathrm{pH}$ values in water and $\mathrm{KCl}$, in the same depths, were lower than those observed for the ADE soils, except for some points of ADE 1, ranging from 4.26 to 6.18 and from 4.01 and 5.88 (Table IV), respectively.

The higher $\mathrm{pH}$ values in water and $\mathrm{KCl}$ for $\mathrm{ADE}$ soils are due to the larger amount of plant and animal residues introduced during its formation [27]. The addiction of these residues results in the incorporation of high levels of exchangeable $\mathrm{Ca}^{2+}$ and $\mathrm{Mg}^{2+}$, typically higher than 100 and $30 \mathrm{mmol} \mathrm{kg}^{-1}$, respectively [27-28].

In general, ADE 1 presented higher $\mathrm{pH}$ values in comparison with ADE 2 (Tables II and III), at the same depths. This was probably due to the higher incorporation of residues containing calcium carbonate in soil (e.g. fish bones and carapaces of turtles), and may be related to the population at that site have been larger than the ADE 2 [29-30]. The $\triangle \mathrm{pH}$ values in the soil layers at both sites and in the adjacent area presented negative values (Tables II-IV). The increase in soil negative charges in these layers enhances the adsorption of cationic elements such as $\mathrm{Ca}^{2+}, \mathrm{Mg}^{2+}$ and $\mathrm{K}^{+}$which is normally desirable [31]. 
The content of the exchangeable cations $\left(\mathrm{Ca}^{2+}\right.$ and $\left.\mathrm{Mg}^{2+}\right)$ were higher in anthropogenic soil than in nonanthropogenic soil (Tables II-IV). The high $\mathrm{Ca}^{2+}$ concentrations in anthropogenic soils are mainly associated with the human and animal bones input [9,32-33]. Furthermore, $\mathrm{Ca}^{2+}$ can form highly stable organometallic complexes, mainly associated with pyrogenic carbon [27,32-33]. At higher depths in ADE 1 and ADE 2, Ca ${ }^{2+}$ and $\mathrm{Mg}^{2+}$ levels were the lowest (Tables II and III), which reflects the absence of leaves and vegetable waste, as well as ceramic fragments, remnants visible bones or charcoal. According to Kern et al., palm leaves used to cover housing, are renewed regularly, and may be an important source of $\mathrm{Mg}^{2+}$. In addition, higher $\mathrm{Mg}^{2+}$ levels in the soil surface may be directly related to the recent incorporation of plant remains (e.g. leaves) [34].

The $\mathrm{K}^{+}$amounts associated with anthropogenic soils, in general, were low $\left(<1.1 \mathrm{mmol} \mathrm{kg}^{-1}\right)$, and this may indicate that the discarded waste had low concentration of this nutrient and/or the high rainfall precipitation in the Amazon region which increased the $\mathrm{K}^{+}$losses by leaching [35]. Nevertheless, high amounts of $\mathrm{K}^{+}\left(>2.3 \mathrm{mmol} \mathrm{kg}{ }^{-1}\right)$ were observed in ADE 2 (Table III), where the maximum amounts at point 4 were $6.1(0-20 \mathrm{~cm})$ and $5.9 \mathrm{mmol} \mathrm{kg}^{-1}(20-40 \mathrm{~cm})$. In ADE 1, the highest $\mathrm{K}^{+}$concentration was $1.6 \mathrm{mmol}$ $\mathrm{kg}^{-1}$ (point 1 at 0-20 cm) (Table II), interpreted as average (from 1.1 to $2.3 \mathrm{mmol} \mathrm{kg}{ }^{-1}$ ) [36].

Some studies on chemical characterization of ADE soils have shown that $\mathrm{K}^{+}$levels are lower than the $\mathrm{P}$, $\mathrm{Ca}^{2+}$ and $\mathrm{Mg}^{2+}$. Falcão and Borges reported values of 1.9 and $1.2 \mathrm{mmol} \mathrm{kg}^{-1}$ in ADE fertilized and nonfertilized, respectively, from Iranduba, AM, Brazil [14]. These results show that ADE exhibits an average content of exchangeable $\mathrm{K}^{+}$very close to the lower limit of the range considered appropriate for most crops. In this way, supplementary potassium fertilization is necessary in order to obtain satisfactory production.

The $\mathrm{K}^{+}$amounts are generally low in ADE, although still higher than those observed in non-anthropogenic areas. Moreover, the higher $\mathrm{K}^{+}$levels observed in adjacent soil surfaces may be related to the recent incorporation of plant residues. This may be associated with higher incorporation of residues containing potassium (leaves and vegetable residues) in $\mathrm{ADE}$, or even cycling $\mathrm{K}^{+}$in plants, which can be absorbed by the roots or even incorporated in their tissues [37].

The exchangeable aluminum values $\left(\mathrm{Al}^{3+}\right)$ were low $\left(<2.0 \mathrm{mmol} \mathrm{kg}^{-1}\right)$ in all depths of ADEs and nonanthropogenic soils, with higher levels observed in the latter (Tables II-IV). This occurred probably because of the considerable amounts of exchangeable bases, notably in ADE sites, corroborating the observation of Brasil and Cravo [36]. These results show that ADEs offer no problems with toxicity of $A l^{3+}$ to plants [5]. 


\section{Article}

Table II. Chemical characteristics of the ADE 1

\begin{tabular}{|c|c|c|c|c|c|c|c|c|c|}
\hline Depth (cm) & $\mathrm{pH}\left(\mathrm{H}_{2} \mathrm{O}\right)$ & $\mathrm{pH}(\mathrm{KCl})$ & $\Delta \mathrm{pH}$ & $\begin{array}{c}P_{\text {org }} \\
\left(\mathrm{mg} \mathrm{kg}^{-1}\right)\end{array}$ & $\begin{array}{c}\mathbf{P}_{\mathrm{ava}} \\
\left(\mathrm{mg} \mathrm{kg}^{-1}\right)\end{array}$ & $\begin{array}{c}\mathrm{Ca}^{2+} \\
\left(\mathrm{mmol} \mathrm{kg}^{-1}\right)\end{array}$ & $\begin{array}{c}\mathrm{Mg}^{2+} \\
\left(\mathrm{mmol} \mathrm{kg}^{-1}\right)\end{array}$ & $\begin{array}{c}\mathrm{K}^{+} \\
\left(\mathrm{mmol} \mathrm{kg}^{-1}\right)\end{array}$ & $\begin{array}{c}\mathrm{Al}^{3+} \\
\left(\mathrm{mmol} \mathrm{kg}^{-1}\right)\end{array}$ \\
\hline \multicolumn{10}{|l|}{ Collect point 1} \\
\hline (1) $0-20$ & $6.05 \pm 0.03$ & $5.26 \pm 0.02$ & -0.79 & $159 \pm 10$ & $233 \pm 11$ & $2242 \pm 34$ & $69.1 \pm 1.9$ & $1.6 \pm 0.16$ & $\mathrm{Nd}$ \\
\hline (2) $20-40$ & $5.48 \pm 0.02$ & $4.85 \pm 0.02$ & -0.63 & $58 \pm 8$ & $842 \pm 12$ & $1812 \pm 30$ & $38.3 \pm 0.9$ & $0.4 \pm 0.05$ & $0.1 \pm 0.05$ \\
\hline (3) $40-60$ & $5.86 \pm 0.03$ & $5.04 \pm 0.02$ & -0.82 & $86 \pm 8$ & $738 \pm 13$ & $1770 \pm 27$ & $32.6 \pm 0.7$ & $0.3 \pm 0.05$ & $\mathrm{Nd}$ \\
\hline (4) $60-80$ & $5.83 \pm 0.03$ & $5.13 \pm 0.02$ & -0.70 & $90 \pm 6$ & $332 \pm 14$ & $1199 \pm 20$ & $33.7 \pm 0.8$ & $0.1 \pm 0.05$ & $\mathrm{Nd}$ \\
\hline (5) $80-100$ & $5.94 \pm 0.03$ & $5.19 \pm 0.02$ & -0.75 & $128 \pm 9$ & $565 \pm 15$ & $843 \pm 20$ & $32.6 \pm 0.8$ & $0.8 \pm 0.10$ & $\mathrm{Nd}$ \\
\hline \multicolumn{10}{|l|}{ Collect point 2} \\
\hline (6) $0-20$ & $7.72 \pm 0.04$ & $6.61 \pm 0.03$ & $-1,11$ & $1412 \pm 61$ & $672 \pm 12$ & $1550 \pm 37$ & $\mathrm{Nd}$ & $0.2 \pm 0.05$ & $\mathrm{Nd}$ \\
\hline (7) $20-40$ & $7.29 \pm 0.03$ & $6.00 \pm 0.03$ & -1.29 & $722 \pm 13$ & $691 \pm 10$ & $1859 \pm 38$ & $\mathrm{Nd}$ & $0.1 \pm 0.05$ & $\mathrm{Nd}$ \\
\hline (8) $40-60$ & $5.82 \pm 0.03$ & $4.94 \pm 0.02$ & -0.88 & $246 \pm 8$ & $228 \pm 8$ & $1424 \pm 27$ & $\mathrm{Nd}$ & $0.1 \pm 0.05$ & $0.1 \pm 0.05$ \\
\hline (9) 60-80 & $5.73 \pm 0.03$ & $4.84 \pm 0.02$ & -0.89 & $55 \pm 7$ & $608 \pm 10$ & $769 \pm 18$ & $\mathrm{Nd}$ & $0.1 \pm 0.05$ & $0.1 \pm 0.05$ \\
\hline (10) $80-100$ & $8.22 \pm 0.07$ & $6.70 \pm 0.03$ & -1.52 & $281 \pm 13$ & $676 \pm 11$ & $465 \pm 6$ & $\mathrm{Nd}$ & $0.8 \pm 0.11$ & $\mathrm{Nd}$ \\
\hline \multicolumn{10}{|l|}{ Collect point 3} \\
\hline (11) $0-20$ & $5.04 \pm 0.02$ & $4.87 \pm 0.02$ & -0.17 & $459 \pm 11$ & $1327 \pm 19$ & $3352 \pm 44$ & $12.0 \pm 0.5$ & $0.5 \pm 0.10$ & $0.1 \pm 0.05$ \\
\hline (12) $20-40$ & $5.08 \pm 0.02$ & $4.89 \pm 0.03$ & -0.19 & $651 \pm 11$ & $2744 \pm 60$ & $2242 \pm 38$ & $\mathrm{Nd}$ & $0.2 \pm 0.05$ & $0.1 \pm 0.05$ \\
\hline (13) $40-60$ & $5.45 \pm 0.03$ & $5.02 \pm 0.03$ & -0.43 & $41 \pm 7$ & $2544 \pm 49$ & $1775 \pm 32$ & $\mathrm{Nd}$ & $0.2 \pm 0.05$ & $\mathrm{Nd}$ \\
\hline (14) 60-80 & $5.96 \pm 0.03$ & $5.09 \pm 0.02$ & -0.87 & $530 \pm 12$ & $1172 \pm 35$ & $1576 \pm 33$ & $\mathrm{Nd}$ & $0.1 \pm 0.04$ & $\mathrm{Nd}$ \\
\hline (15) 80-100 & $6.09 \pm 0.03$ & $5.17 \pm 0.02$ & -0.92 & $135 \pm 10$ & $204 \pm 10$ & $1021 \pm 14$ & $\mathrm{Nd}$ & $1.6 \pm 0.12$ & $\mathrm{Nd}$ \\
\hline \multicolumn{10}{|c|}{ Collect point 4} \\
\hline (16) $0-20$ & $5.44 \pm 0.03$ & $4.71 \pm 0.04$ & -0.73 & $356 \pm 10$ & $1138 \pm 44$ & $1445 \pm 17$ & $24.6 \pm 0.6$ & $0.3 \pm 0.04$ & $0.2 \pm 0.05$ \\
\hline (17) $20-40$ & $5.78 \pm 0.03$ & $4.93 \pm 0.03$ & -0.85 & $56 \pm 6$ & $598 \pm 16$ & $2105 \pm 35$ & $36.0 \pm 0.8$ & $0.2 \pm 0.05$ & $\mathrm{Nd}$ \\
\hline (18) 40-60 & $5.95 \pm 0.02$ & $5.08 \pm 0.02$ & -0.87 & $235 \pm 10$ & $250 \pm 9$ & $1162 \pm 19$ & $32.7 \pm 0.8$ & $0.1 \pm 0.04$ & $\mathrm{Nd}$ \\
\hline (19) 60-80 & $5.99 \pm 0.04$ & $5.18 \pm 0.03$ & -0.81 & $19 \pm 2$ & $230 \pm 8$ & $911 \pm 11$ & $\mathrm{Nd}$ & $0.1 \pm 0.04$ & $\mathrm{Nd}$ \\
\hline (20) 80-100 & $5.58 \pm 0.02$ & $5.83 \pm 0.02$ & -0.25 & $153 \pm 7$ & $449 \pm 12$ & $670 \pm 5$ & $\mathrm{Nd}$ & $0.8 \pm 0.06$ & $\mathrm{Nd}$ \\
\hline \multicolumn{10}{|l|}{ Collect point 5} \\
\hline (21) 0-20 & $7.28 \pm 0.05$ & $6.63 \pm 0.04$ & -0.65 & $277 \pm 8$ & $785 \pm 4$ & $2242 \pm 49$ & $10.9 \pm 0.4$ & $0.2 \pm 0.05$ & $\mathrm{Nd}$ \\
\hline (22) $20-40$ & $7.21 \pm 0.04$ & $6.11 \pm 0.04$ & -1.10 & $483 \pm 9$ & $904 \pm 8$ & $1398 \pm 28$ & $\mathrm{Nd}$ & $0.3 \pm 0.05$ & $\mathrm{Nd}$ \\
\hline (23) 40-60 & $7.26 \pm 0.04$ & $6.41 \pm 0.03$ & -0.85 & $323 \pm 9$ & $316 \pm 6$ & $1660 \pm 28$ & $\mathrm{Nd}$ & $0.2 \pm 0.05$ & $\mathrm{Nd}$ \\
\hline (24) 60-80 & $7.62 \pm 0.04$ & $6.44 \pm 0.02$ & -1.85 & $417 \pm 5$ & $367 \pm 12$ & $685 \pm 14$ & $\mathrm{Nd}$ & $\mathrm{Nd}$ & $\mathrm{Nd}$ \\
\hline (25) 80-100 & $5.43 \pm 0.02$ & $4.62 \pm 0.03$ & -0.81 & $116 \pm 7$ & $828 \pm 14$ & $1000 \pm 18$ & $\mathrm{Nd}$ & $0.4 \pm 0.04$ & $0.3 \pm 0.06$ \\
\hline
\end{tabular}


Table III. Chemical characteristics of the ADE 2

\begin{tabular}{|c|c|c|c|c|c|c|c|c|c|}
\hline Depth (cm) & $\mathrm{pH}\left(\mathrm{H}_{2} \mathrm{O}\right)$ & $\mathrm{pH}(\mathrm{KCl})$ & $\Delta \mathrm{pH}$ & $\begin{array}{c}P_{\text {org }} \\
\left(\mathrm{mg} \mathrm{kg}^{-1}\right)\end{array}$ & $\underset{\left(\mathbf{m g ~ k g}^{-1}\right)}{P_{\text {ava }}}$ & $\begin{array}{c}\mathrm{Ca}^{2+} \\
\left(\mathrm{mmol} \mathrm{kg}^{-1}\right)\end{array}$ & $\begin{array}{c}\mathrm{Mg}^{2+} \\
\left(\mathrm{mmol} \mathrm{kg}^{-1}\right)\end{array}$ & $\begin{array}{c}\mathrm{K}^{+} \\
\left(\mathrm{mmol}_{\mathrm{c}} \mathrm{kg}^{-1}\right)\end{array}$ & $\begin{array}{c}\mathrm{Al}^{3+} \\
\left(\mathrm{mmol} \mathrm{kg}^{-1}\right)\end{array}$ \\
\hline \multicolumn{10}{|c|}{ Collect point 1} \\
\hline (26) 0-20 & $5.85 \pm 0.01$ & $5.38 \pm 0.03$ & -0.47 & $197 \pm 12$ & $967 \pm 14$ & $1220 \pm 28$ & $41.7 \pm 1.0$ & $0.8 \pm 0.05$ & $\mathrm{Nd}$ \\
\hline (27) $20-40$ & $5.82 \pm 0.03$ & $5.03 \pm 0.04$ & -0.79 & $107 \pm 8$ & $26 \pm 9$ & $911 \pm 11$ & $42.8 \pm 1.1$ & $0.7 \pm 0.06$ & $0.1 \pm 0.04$ \\
\hline (28) 40-60 & $5.53 \pm 0.04$ & $4.60 \pm 0.03$ & -0.93 & $31 \pm 4$ & $951 \pm 13$ & $586 \pm 7$ & $46.3 \pm 1.1$ & $0.2 \pm 0.04$ & $0.3 \pm 0.05$ \\
\hline (29) 60-80 & $5.47 \pm 0.03$ & $4.49 \pm 0.02$ & -0.98 & $38 \pm 4$ & $812 \pm 7$ & $397 \pm 4$ & $49.7 \pm 1.6$ & $0.4 \pm 0.05$ & $0.4 \pm 0.05$ \\
\hline (30) 80-100 & $5.42 \pm 0.02$ & $4.28 \pm 0.03$ & -1.14 & $60 \pm 5$ & $538 \pm 8$ & $172 \pm 3$ & $47.4 \pm 1.5$ & $0.3 \pm 0.06$ & $0.1 \pm 0.05$ \\
\hline \multicolumn{10}{|l|}{ Collect point 2} \\
\hline (31) 0-20 & $5.45 \pm 0.04$ & $4.49 \pm 0.03$ & -0.96 & $67 \pm 5$ & $234 \pm 10$ & $1508 \pm 36$ & $46.0 \pm 1.8$ & $2.0 \pm 0.23$ & $0.2 \pm 0.05$ \\
\hline (32) $20-40$ & $5.01 \pm 0.02$ & $3.76 \pm 0.05$ & -1.25 & $77 \pm 8$ & $255 \pm 5$ & $465 \pm 10$ & $47.4 \pm 1.7$ & $4.8 \pm 0.34$ & $1.2 \pm 0.12$ \\
\hline (33) 40-60 & $4.68 \pm 0.03$ & $3.70 \pm 0.06$ & -0.98 & $68 \pm 6$ & $250 \pm 7$ & $130 \pm 3$ & $36.0 \pm 1.5$ & $1.9 \pm 0.19$ & $1.1 \pm 0.10$ \\
\hline (34) 60-80 & $4.97 \pm 0.03$ & $3.92 \pm 0.06$ & -1.05 & $220 \pm 9$ & $978 \pm 10$ & $8.9 \pm 0.3$ & $41.7 \pm 1.3$ & $3.4 \pm 0.26$ & $0.7 \pm 0.04$ \\
\hline (35) $80-100$ & $5.22 \pm 0.02$ & $4.13 \pm 0.02$ & -1.09 & $43 \pm 6$ & $946 \pm 10$ & $560 \pm 10$ & $45.1 \pm 1.6$ & $4.5 \pm 0.28$ & $0.3 \pm 0.05$ \\
\hline \multicolumn{10}{|l|}{ Collect point 3} \\
\hline (36) $0-20$ & $5.48 \pm 0.02$ & $4.66 \pm 0.03$ & -0.82 & $233 \pm 7$ & $1238 \pm 16$ & $2194 \pm 43$ & $53.1 \pm 2.3$ & $2.4 \pm 0.15$ & $0.4 \pm 0.05$ \\
\hline (37) 20-40 & $5.45 \pm 0.02$ & $4.47 \pm 0.02$ & -0.98 & $132 \pm 6$ & $980 \pm 8$ & $1086 \pm 23$ & $47.4 \pm 1.5$ & $1.8 \pm 0.09$ & $0.2 \pm 0.04$ \\
\hline (38) 40-60 & $5.45 \pm 0.03$ & $4.53 \pm 0.02$ & -0.92 & $158 \pm 8$ & $418 \pm 8$ & $1891 \pm 38$ & $46.3 \pm 1.4$ & $1.1 \pm 0.07$ & $0.2 \pm 0.05$ \\
\hline (39) 60-80 & $5.79 \pm 0.02$ & $4.92 \pm 0.03$ & -0.87 & $258 \pm 5$ & $720 \pm 7$ & $1848 \pm 37$ & $42.8 \pm 1.4$ & $0.6 \pm 0.05$ & $\mathrm{Nd}$ \\
\hline (40) $80-100$ & $5.89 \pm 0.03$ & $5.13 \pm 0.03$ & -0.76 & $58 \pm 6$ & $441 \pm 13$ & $1277 \pm 30$ & $39.4 \pm 1.3$ & $0.6 \pm 0.03$ & $\mathrm{Nd}$ \\
\hline \multicolumn{10}{|l|}{ Collect point 4} \\
\hline (41) 0-20 & $6.12 \pm 0.03$ & $5.38 \pm 0.03$ & -0.74 & $294 \pm 7$ & $309 \pm 7$ & $2373 \pm 59$ & $130.8 \pm 3.0$ & $6.1 \pm 0.35$ & $0.1 \pm 0.06$ \\
\hline (42) $20-40$ & $4.82 \pm 0.03$ & $3.73 \pm 0.04$ & -1.09 & $90 \pm 9$ & $213 \pm 5$ & $712 \pm 20$ & $53.1 \pm 1.3$ & $5.9 \pm 0.30$ & $1.1 \pm 0.10$ \\
\hline (43) $40-60$ & $4.57 \pm 0.02$ & $3.53 \pm 0.06$ & -1.04 & $94 \pm 7$ & $210 \pm 6$ & $224 \pm 4$ & $36.0 \pm 0.9$ & $1.6 \pm 0.14$ & $1.6 \pm 0.11$ \\
\hline (44) 60-80 & $4.56 \pm 0.04$ & $3.60 \pm 0.05$ & -0.96 & $37 \pm 3$ & $863 \pm 11$ & $88 \pm 2$ & $33.7 \pm 0.8$ & $1.2 \pm 0.09$ & $1.4 \pm 0.08$ \\
\hline (45) $80-100$ & $4.68 \pm 0.02$ & $3.72 \pm 0.06$ & -0.96 & $47 \pm 3$ & $528 \pm 8$ & $83 \pm 2$ & $33.7 \pm 0.9$ & $1.1 \pm 0.12$ & $1.1 \pm 0.12$ \\
\hline \multicolumn{10}{|l|}{ Collect point 5} \\
\hline (46) $0-20$ & $5.58 \pm 0.03$ & $4.73 \pm 0.03$ & -0.85 & $1352 \pm 22$ & $399 \pm 5$ & $3876 \pm 93$ & $80.5 \pm 1.9$ & $3.6 \pm 0.26$ & $0.1 \pm 0.05$ \\
\hline (47) $20-40$ & $5.42 \pm 0.03$ & $4.66 \pm 0.03$ & -0.76 & $159 \pm 6$ & $243 \pm 5$ & $1953 \pm 42$ & $42.8 \pm 1.2$ & $1.0 \pm 0.08$ & $0.1 \pm 0.04$ \\
\hline (48) $40-60$ & $5.30 \pm 0.03$ & $4.45 \pm 0.04$ & -0.85 & $154 \pm 6$ & $871 \pm 8$ & $1162 \pm 22$ & $41.7 \pm 1.1$ & $0.5 \pm 0.05$ & $0.3 \pm 0.05$ \\
\hline (49) 60-80 & $5.01 \pm 0.02$ & $4.09 \pm 0.03$ & -0.92 & $62 \pm 4$ & $242 \pm 7$ & $743 \pm 21$ & $38.3 \pm 1.0$ & $0.3 \pm 0.06$ & $0.6 \pm 0.06$ \\
\hline (50) $80-100$ & $5.09 \pm 0.03$ & $4.04 \pm 0.04$ & -1.05 & $34 \pm 3$ & $1250 \pm 21$ & $467 \pm 10$ & $38.3 \pm 1.1$ & $0.5 \pm 0.07$ & $0.5 \pm 0.05$ \\
\hline
\end{tabular}

Nd: Below Limit of Detection 


\section{Article}

Table IV. Chemical characteristics of the non-anthropogenic soil

\begin{tabular}{|c|c|c|c|c|c|c|c|c|c|}
\hline Depth (cm) & $\mathrm{pH}\left(\mathrm{H}_{2} \mathrm{O}\right)$ & $\mathrm{pH}(\mathrm{KCl})$ & $\Delta \mathrm{pH}$ & $\begin{array}{c}P_{\text {org }} \\
\left(\mathrm{mg} \mathrm{kg}^{-1}\right)\end{array}$ & $\begin{array}{c}P_{\text {ava }} \\
\left(\mathrm{mg} \mathrm{kg}^{-1}\right)\end{array}$ & $\begin{array}{c}\mathrm{Ca}^{2+} \\
\left(\mathrm{mmol} \mathrm{kg}^{-1}\right)\end{array}$ & $\begin{array}{c}\mathrm{Mg}^{2+} \\
\left(\mathrm{mmol} \mathrm{kg}^{-1}\right)\end{array}$ & $\begin{array}{c}\mathrm{K}^{+} \\
\left(\mathrm{mmol} \mathrm{kg}{ }^{-1}\right)\end{array}$ & $\begin{array}{c}\mathrm{Al}^{3+} \\
\left(\mathrm{mmol} \mathrm{kg}^{-1}\right)\end{array}$ \\
\hline \multicolumn{10}{|c|}{ Collect point 1} \\
\hline (52) 20-40 & $4.58 \pm 0.03$ & $4.14 \pm 0.05$ & -0.44 & $55 \pm 4$ & $10 \pm 1$ & $376 \pm 8$ & $\mathrm{Nd}$ & $0.6 \pm 0.05$ & $1.0 \pm 0.11$ \\
\hline (54) 60-80 & $4.57 \pm 0.02$ & $4.15 \pm 0.06$ & -0.42 & $50 \pm 6$ & $9 \pm 1$ & $261 \pm 6$ & $\mathrm{Nd}$ & $0.4 \pm 0.05$ & $1.0 \pm 0.01$ \\
\hline (55) $80-100$ & $4.96 \pm 0.02$ & $4.15 \pm 0.05$ & -0.81 & $62 \pm 6$ & $15 \pm 1$ & $151 \pm 4$ & $\mathrm{Nd}$ & $0.4 \pm 0.05$ & $1.1 \pm 0.12$ \\
\hline \multicolumn{10}{|c|}{ Collect point 2} \\
\hline (56) $0-20$ & $4.99 \pm 0.03$ & $4.07 \pm 0.06$ & -0.92 & $52 \pm 3$ & $9 \pm 1$ & $156 \pm 3$ & $\mathrm{Nd}$ & $0.2 \pm 0.04$ & $0.9 \pm 0.12$ \\
\hline (59) 60-80 & $4.26 \pm 0.03$ & $4.11 \pm 0.05$ & -0.15 & $28 \pm 3$ & $6 \pm 1$ & $99 \pm 2$ & $\mathrm{Nd}$ & $0.2 \pm 0.05$ & $1.0 \pm 0.6$ \\
\hline (60) $80-100$ & $4.33 \pm 0.04$ & $4.13 \pm 0.05$ & -0.20 & $28 \pm 2$ & $7 \pm 1$ & $4 \pm 1$ & $\mathrm{Nd}$ & $0.1 \pm 0.03$ & $1.0 \pm 0.5$ \\
\hline \multicolumn{10}{|c|}{ Collect point 3} \\
\hline (61) $0-20$ & $5.38 \pm 0.03$ & $4.74 \pm 0.04$ & -0.64 & $112 \pm 4$ & $23 \pm 2$ & $1765 \pm 46$ & $46.3 \pm 1.4$ & $3.0 \pm 0.18$ & $\mathrm{Nd}$ \\
\hline (62) $20-40$ & $5.52 \pm 0.03$ & $5.08 \pm 0.03$ & 0.44 & $92 \pm 3$ & $18 \pm 1$ & $979 \pm 25$ & $14.3 \pm 0.3$ & $3.1 \pm 0.23$ & $\mathrm{Nd}$ \\
\hline (63) $40-60$ & $5.32 \pm 0.03$ & $4.98 \pm 0.04$ & -0.34 & $101 \pm 6$ & $14 \pm 1$ & $387 \pm 10$ & $\mathrm{Nd}$ & $0.2 \pm 0.05$ & $0.2 \pm 0.05$ \\
\hline (64) 60-80 & $5.81 \pm 0.02$ & $4.73 \pm 0.04$ & -1.08 & $54 \pm 4$ & $12 \pm 1$ & $313 \pm 11$ & $\mathrm{Nd}$ & $0.2 \pm 0.04$ & $0.2 \pm 0.05$ \\
\hline (69) 60-80 & $5.51 \pm 0.02$ & $4.52 \pm 0.04$ & -0.99 & $52 \pm 5$ & $3 \pm 0.4$ & $\mathrm{Nd}$ & $\mathrm{Nd}$ & $0.1 \pm 0.06$ & $0.5 \pm 0.05$ \\
\hline (70) 80-100 & $5.01 \pm 0.03$ & $4.20 \pm 0.04$ & -0.81 & $41 \pm 4$ & $2 \pm 0.4$ & $\mathrm{Nd}$ & $\mathrm{Nd}$ & $0.1 \pm 0.04$ & $0.2 \pm 0.04$ \\
\hline \multicolumn{10}{|c|}{ Collect point 5} \\
\hline (71) $0-20$ & $4.88 \pm 0.03$ & $4.10 \pm 0.04$ & -0.78 & $128 \pm 7$ & $19 \pm 1$ & $347 \pm 8$ & $8.1 \pm 0.3$ & $0.2 \pm 0.06$ & $\mathrm{Nd}$ \\
\hline (72) $20-40$ & $4.56 \pm 0.03$ & $4.15 \pm 0.06$ & -0.41 & $38 \pm 3$ & $10 \pm 1$ & $283 \pm 8$ & $2.3 \pm 0.1$ & $0.1 \pm 0.05$ & $\mathrm{Nd}$ \\
\hline (73) 40-60 & $4.76 \pm 0.03$ & $4.05 \pm 0.04$ & -0.71 & $27 \pm 0.4$ & $6 \pm 1$ & $192 \pm 4$ & $\mathrm{Nd}$ & $0.2 \pm 0.05$ & $0.3 \pm 0.05$ \\
\hline (74) 60-80 & $4.82 \pm 0.02$ & $4.07 \pm 0.05$ & -0.75 & $20 . \pm 1$ & $4 \pm 1$ & $167 \pm 3$ & $\mathrm{Nd}$ & $0.1 \pm 0.05$ & $0.2 \pm 0.05$ \\
\hline (75) 80-100 & $4.78 \pm 0.03$ & $4.11 \pm 0.04$ & $-0,67$ & $8 \pm 1$ & $2 \pm 1$ & $146 \pm 4$ & $\mathrm{Nd}$ & $0.1 \pm 0.04$ & $0.2 \pm 0.04$ \\
\hline
\end{tabular}

Nd: Below Limit of Detection 
High levels of $P_{\text {ava }}$ were observed in all depths of both ADEs, with the highest levels observed for ADE 1 (Tables II and III). The minimum and maximum amounts of $P_{\text {ava }}$ ranged from $204 \mathrm{mg} \mathrm{kg}^{-1}(20-40 \mathrm{~cm})$ to 2740 $\mathrm{mg} \mathrm{kg}^{-1}(80-100 \mathrm{~cm})$ in point 3 (Table II), whereas ADE 2, the minimum and maximum $P_{\text {ava }}$ values were 213 $\mathrm{mg} \mathrm{kg}^{-1}$ (point 4, depth 20-40 cm) and $1250 \mathrm{mg} \mathrm{kg}^{-1}$ (point 5, depth 80-100 cm), respectively (Table III). On the other hand, in non-anthropogenic soil the maximum amount was $22.6 \mathrm{mg} \mathrm{kg}^{-1}$ (point 3, depth 0-20 cm), which was around 121 and 55 times lower than the maximum amount found, respectively, in ADE 1 and ADE 2 (Table IV). These higher levels of available phosphorus in the anthropogenic layers are due to the high incorporation of residues, mainly animal remains [38].

The maximum amounts of $P_{\text {org }}$ were $1410 \mathrm{mg} \mathrm{kg}^{-1}$ (point 1, depth 0-20 cm) (Table II) and $1350 \mathrm{mg} \mathrm{kg}^{-1}$ (point 5, depth 0-20 cm) (Table III), respectively, in ADE 1 and ADE 2, while the value in the nonanthropogenic soil was $222 \mathrm{mg} \mathrm{kg}^{-1}$ (point 1, depth 0-20 cm) (Table IV). Fraser and Clement evaluated ADE in the Amazonic region and attributed these high $\mathrm{P}_{\text {org }}$ and $\mathrm{P}_{\text {ava }}$ values to the addition of animal bones by preColumbian people [38].

Regarding variance analysis, the first component explained $45.1 \%$ and $47.7 \%$, and the second component explained $20 \%$ and $22.8 \%$ of the variance among the investigated parameters in soil samples, respectively, from the ADE 1 and non-anthropogenic soil; and between ADE 2 and non-anthropogenic soil (Figures $2 \mathrm{a}$ and $\mathrm{b}$ ). The points referring to the non-anthropogenic area were not weighted in one of the parameters that compose each principal component, according to the simultaneous analysis involving scores and loadings. This shows that these parameters did not show high variability in these points, nor enough correlation to be accountable for a group formation (Figure 2).

$\mathrm{P}_{\text {org }}, \mathrm{P}_{\text {ava }}, \mathrm{pH}$ values in water and $\mathrm{KCl}$ had potential to discriminate ADE 1 from adjacent soil, once some non-anthropogenic soils were observed in this group. The remaining ADE 1 samples formed a distinct group from $\mathrm{Mg}^{2+}, \mathrm{K}^{+}$and $\mathrm{Al}^{3+}$. The higher weight to the formation of this group was assigned to the $\mathrm{Mg}^{2+}$ amount (Figure 2A and 2B).

The $\mathrm{P}_{\text {ava }}$ and $\mathrm{Ca}^{2+}$ had the highest correlations at the ADE 1 and $\mathrm{ADE} 2$, and $\mathrm{pH}$ in water and $\mathrm{KCl}, \mathrm{Ca}^{2+}$, $\mathrm{P}_{\text {org }}$ and $\mathrm{P}_{\text {ava }}$ had a positive correlation (Figure $2 \mathrm{~F}$ ). This high correlation is associated with the incorporation of human and animal bone remains [32-33]. Furthermore, $\mathrm{Al}^{3+}, \mathrm{K}^{+}$and $\mathrm{Mg}^{2+}$ were negatively correlated (Figure 2). In both anthropogenic soils, the $\mathrm{K}^{+}$amount had the least correlation with other attributes. This may have been favored by the low concentrations of this cation, in addition to the fact that the sandy soil texture and water regime favored its leaching in the soil profile [39], since the only valence load is poorly adsorbed on soil colloids [40]. The strongest correlations between ADE 1 and ADE 2 were observed in relation to $\mathrm{pH}$ values in water and in $\mathrm{KCl}$ (Figure $2 \mathrm{~F}$ ). 


\section{Article}
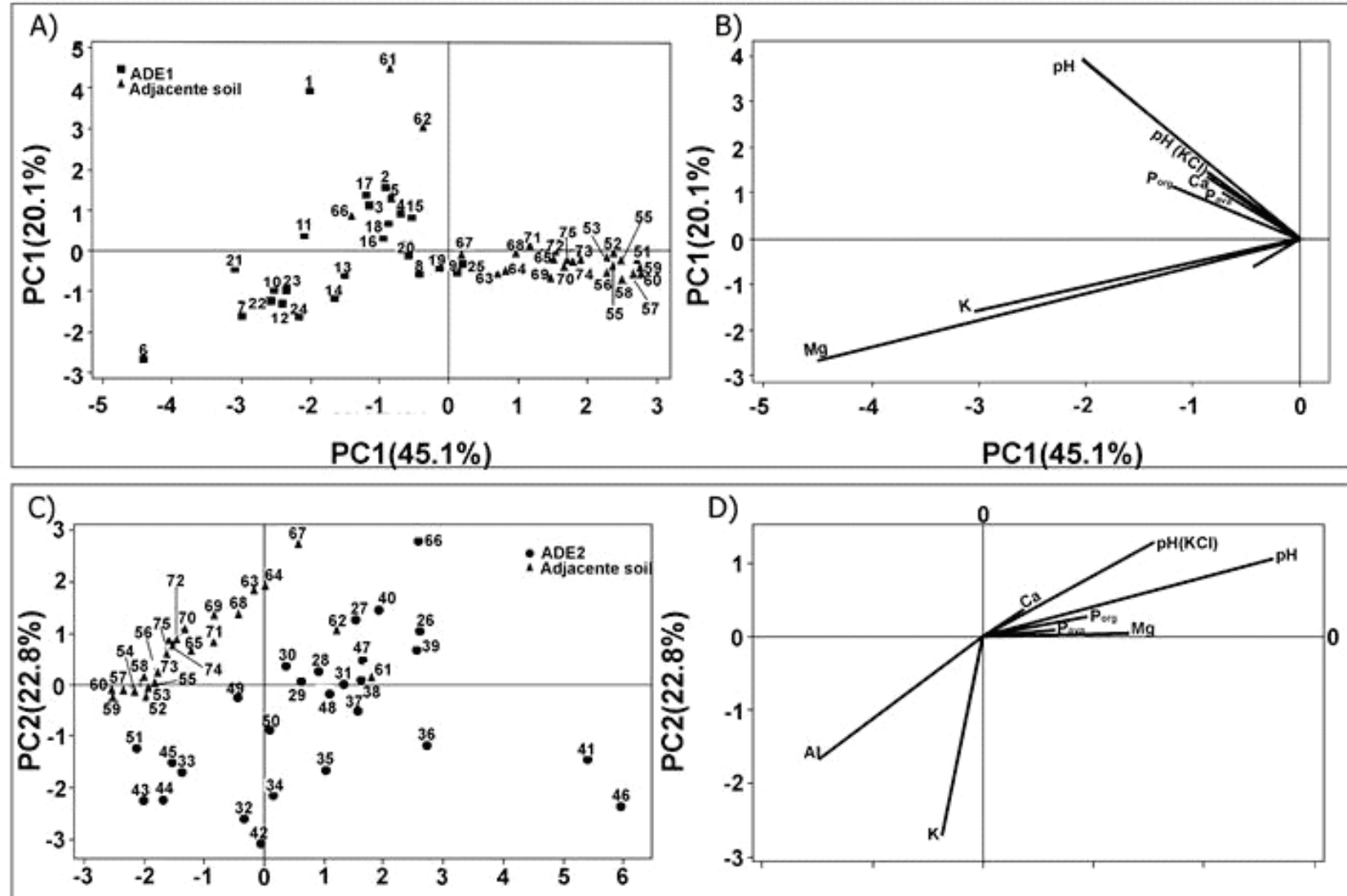

PC1 $(47.7 \%)$

PC1 $(47.7 \%)$
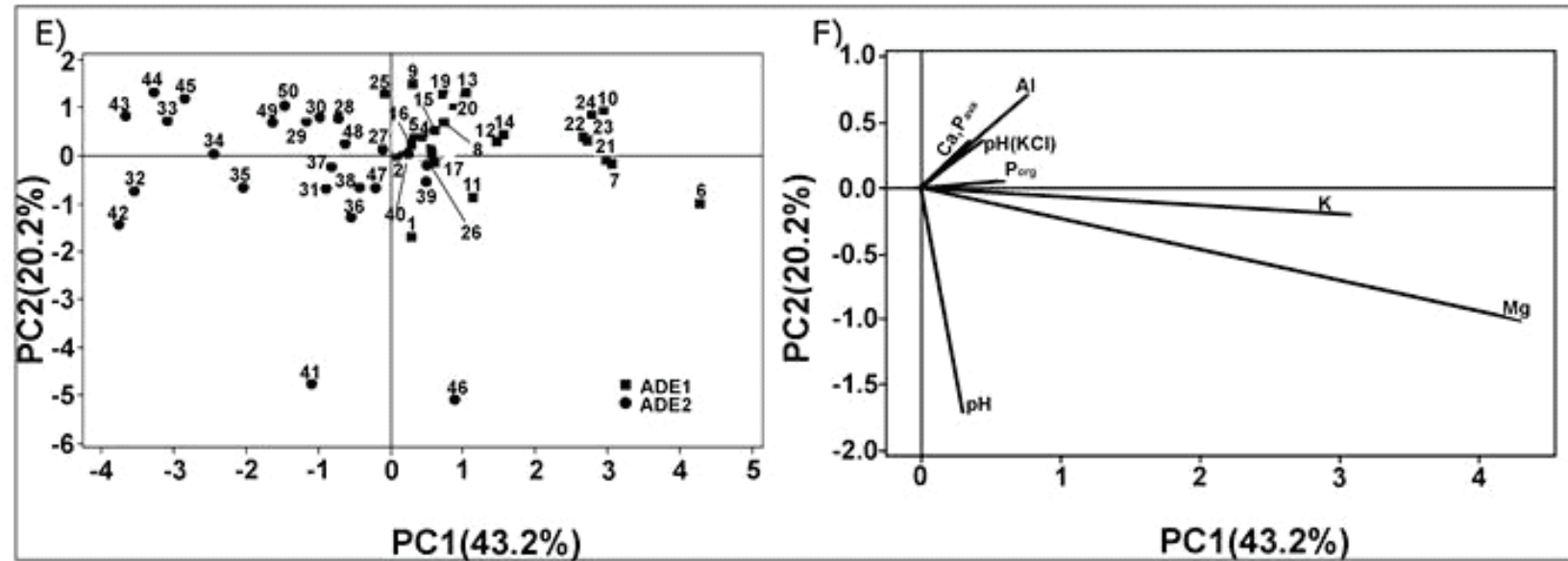

Figure 2. Principal component analysis of the averages of chemical attributes between ADE $1(\boldsymbol{\bullet})$ and non-anthropogenic soil $(\boldsymbol{\Delta})(A$ and $B)$; ADE $2(\bullet)$ and non-anthropogenic soil $(\boldsymbol{\Delta})(C$ e D); and ADE 1 ( $\bullet$ ) and ADE $2(\bullet)(E$ e F). (A, C and E) Scores. (B, D and F) Loadings. Numbers refer to the Tables I, II and III. 


\section{CONCLUSION}

The amount of $\mathrm{P}_{\text {org }}, \mathrm{P}_{\text {ava }}, \mathrm{Ca}^{2+}$ and $\mathrm{Mg}^{2+}$ as well as $\mathrm{pH}$ values in water and in $\mathrm{KCl}$ were higher in $\mathrm{ADE}$ in relation to non-anthropogenic soils, and his aspects gives rise to the soil increased fertility. The low $\mathrm{K}^{+}$ amounts in both ADEs may be limiting for some crops if they not receive additional fertilization with this nutrient. The high amounts of $\mathrm{P}_{\text {org }}$ and $\mathrm{P}_{\text {ava }}$ and the correlation with $\mathrm{Ca}^{2+}$ amount in anthropogenic soils is directly related to bone incorporation, possibly of fish. The $\mathrm{pH}$ values in water and $\mathrm{KCl}$ have the potential to differentiate anthropogenic from non-anthropogenic soils.

\section{ACKNOWLEDGEMENTS}

This work was supported by the Brazilian Federal entities CNPq (National Council for Scientific and Technological Development) and CAPES (Coordination of Improvement of Higher Education Personnel).

\section{Conflict of Interest Statement}

The authors declare no conflict of interest.

Manuscript received: 10/02/18; revised manuscript received: 11/03/18; revised manuscript for the $2^{\text {nd }}$ time received: 11/14/18; manuscript accepted: 11/14/18; published online November 28, 2018.

\section{REFERENCES}

1. Cardoso, E. L.; Silva, M. L. N.; Moreira, F. M. S.; Curi, N. Pesq. Agropec. Bras., 2009, 44, pp 631637 (DOI: 10.1590/S0100-204X2009000600012).

2. Quesada, C. A.; Lloyd, J.; Anderson, L. O.; Fyllas, N. M.; Schwarz, M.; Czimczik, C. I. Biogeosciences, 2001, 8, pp 1415-1440 (DOI: 10.5194/bg-8-1415-2011).

3. Kämpf, N.; Woods, W. I.; Kern, D. C.; Cunha, T. J. F. Classificação das terras pretas de índio e outros solos antrópicos antigos. In: Teixeira, W. G.; Kern, D. C.; Madari, B. E.; Lima, H. N.; Woods, W. (Ed). As Terras Pretas de índio da Amazônia: Sua Caracterização e Uso deste Conhecimento na Criação de Novas Áreas. Embrapa Amazônia Ocidental, Manaus, 2009, pp 87-102.

4. Glaser, B.; Birk, J. J. Geochim. Cosmochim. Acta., 2012, 82, pp 39-51 (DOI: 10.1016/j.gca.2010.11.029).

5. Falcão, N. P. S.; Clement, C. R.; Tsai, S. M.; Comerford, N. B. Pedology, fertility, and biology of central Amazonian Dark Earths. In: Woods W. I. et al. (Ed ). Amazonian Dark Earths: Win Sombroeks Vision. Springer, Berlin, 2009, pp 213-228.

6. Kämpf, N.; Kern, D. C. O solo como registro da ocupação humana pré-histórica na Amazônia. Tópicos em ciência do solo. Viçosa, MG, Sociedade Brasileira de Ciência do Solo, 2005, 4, pp 277-320.

7. Schellenkens, J.; Almeida-Santos, J.; Macedo, R. S.; Buurman, P.; Kuyper, T. W.; Vidal-Torrado, P. Geoderma, 2017, 288, pp 154 -165 (DOI: 10.106/j.geoderma.2016.11.001).

8. Glaser, B. Prehistorically modified soils of central Amazonia: a model for sustainable agriculture in the twenty-first century. Philos. Trans. R. Soc. B; Biol. Sci., 2007, 362, pp 187-196 (DOI: 10.1098/rstb.2006.1978).

9. Schmidt, M. J.; Py-Daniel, A. R.; Moraes, C. P.; Valle, R. B.; Caromano, C. F.; Teixeira, W. G.; Barbosa, C. A.; Fonseca, J. A.; Magalhaes, M. P.; Santos, D. S. C.; Silva, R. S.; Guapindaia, V. L.; Moraes, B.; Lima, H. P.; Neves, E. G.; Heckenberger, M. J. J. Archaeol. Sci., 2014, 42, pp 152-165 (DOI: 10.1016/j.jas.2013.11.002). 


\section{Article}

10. Taube, P. S.; Hansel, F. A.; Madureira, L. A. S.; Teixeira, W. G. Org. Geochem., 2013, 58, pp 96-106 (DOI: 10.1016/j.orggeochem.2013.02.004).

11. Silva, F. W. R.; Lima, H. N.; Teixeira, W. G.; Motta, M. B.; Santana, R. M. Rev. Bras. Cienc. Solo, 2011, 35, pp 673-681 (DOI: 10.1590/S0100-06832011000300002).

12. Heckenberger, M.; Neves, E. G. A. Rev. Anthropol., 2009, 38, pp 251-266 (DOI: 10.1146/annurev-anthro-091908-164310).

13. Dexter, A. R.; Czyz, A. D.; Richard, G.; Reszokowska, A. Geoderma, 2008, 143, pp 243-253 (DOI: 10.1016/j.geoderma.2007.11.010).

14. Falcão, N. P. S.; Borges, L. F. Acta Amazonica, 2006, 36, pp 401-406 (DOI: 10.1590/S0044-59672006000400001).

15. Lima, H. N.; Schaefer, C. E. R.; Mello, J. W. V.; Gilkes, R. J., Kern, J. Geoderma, 2002, 110, pp 1-17 (DOI: 10.1016/S0016-7061(02)00141-6).

16. Falcão, N. P. S.; Comerford, N.; Lehmann, J. Determining nutrient bioavailability of Amazonian Dark Earth soils: methodological challenges. In: Lehmann, J.; Kern, D. C.; Glaser, B.; Woods, W. I. (Ed). Amazonian Dark Earths; origin, properties, managements. Kluwer: Academic Publishers, Dordrecht, 2003, pp 255-270.

17. Campos, M. C. C.; Santos, L. A. C.; Silva, D. M. P.; Mantovanelli, B. C.; Soares, M. D. R. Revista Agro@mbiente, 2012, 6, pp 102-109 (DOI: 10.18227/1982-8470ragro.v6i2.682).

18. Novotny, E. H.; Maia, C. M. B. F.; Carvalho, M. M. T.; Madari, B. E. Rev. Bras. Cienc. Solo, 2015, 39, pp 321-344 (DOI: 10.1590/01000683rbcs20140818).

19. Schaan, D. P., Sacred geographies of ancient Amazonia: historical ecology of social complexity, new frontiers in historical ecology, v. 3. Walnut Creek, CA: Left Coast Press, 2012, p 233.

20. Travassos, D. A. Ocupação indígena na foz do rio Tapajós (3260-960 AP): estudo do sítio Porto de Santarém, baixo Amazonas. Master thesis, 2012, (Mestrado em Antropologia). Instituto de Filosofia e Ciências Humanas, Universidade Federal do Pará, Belém, Brazil, p 246.

21. Rocha, E. J. P.; Souza, E. B.; Santos, F. A. A.; Lopes, M. N.; Santos, D. M.; Rolim, P. A. M.; Neto, B. S.; Maia, I. F. Zoneamento climático: relatório preliminar ZEEA-PA. Belém, SIPAM, 2009.

22. Rodrigues, T. E.; Santos, P.; Oliveira Jr, R. C.; Valente, M. A.; Silva, J. M. L.; Cardoso Jr, E. R. Características dos Solos da área do Planalto de Belterra, município de Santarém, Estado do Pará. Embrapa Amazônia Oriental, Belém, 2001, p 55.

23. Empresa Brasileira de Pesquisa Agropecuária - EMBRAPA. Manual de métodos de análises de solo. $2^{\text {nd }}$ Ed., Rio de Janeiro, RJ, Embrapa Solos, 2011.

24. Tedesco, M. J.; Gianello, C.; Bissani, C. A.; Bohnen, H.; Volkweiss, S. J. Análise de solo, plantas e outros materiais. Porto Alegre, UFRGS/FA/DS, 1995.

25. Olsen, S. R.; Sommers, L. E. Phosphorus. In: Page, A. L.; Miller, R. H.; Keeney, Q. R. Methods of Soil Analysis. Chemical and Microbiological Properties. $2^{\text {nd }}$ Ed., Soil Science Society of America - SSSA, Madison, WI, 1982, pp 403-430.

26. Murphy, J.; Riley, J. P. Anal. Chim. Acta, 1962, 27, pp 31-36 (DOI: 10.1016/S0003-2670(00)88444-5).

27. Falcão, N. P. S.; Comerford, N.; Lehmann, J. Determining Nutrient Bioavailability of Amazonian Dark Earth Soils - Methodological Challenges. In: Lehmann, J. et al. (Ed). Amazonian Dark Earths: Origins, Properties, Management. Boston, Kluwer Academic Publishers, 2003, pp 255-270.

28. Sombroek, W. G.; Ruivo, M. L.; Fearnside, P. M.; Glaser, B.; Lehmann, J. Amazonian Dark Earths as carbon stores and sinks. In.: Lehmann, J. et al. (Ed). Amazonian Dark Earths: origin, properties, and management. Dordrecht, Kluwer Academic Publishers, 2003, pp 125-139.

29. Lehmann, J.; Silva Junior, J. P.; Steiner, C.; Nehls, T.; Zech, W.; Glaser, B. Plant Soil., 2003, 249, pp 343-357 (DOI: 10.1023/A:1022833116184). 
30. Lehmann, J.; Kern, D. C.; Glaser, B.; Woods W. I. Amazonian Dark Earths: origin, properties, management. Kluwer Academic Publisher. Dordrecht, 2003, p 523.

31. Liang, B.; Lehmann, J.; Solomon, D.; Kinyangi, J.; Grossman, J.; O'Neill, B.; Skjemstad, J. O.; Thies, J.; Luizão, F. J.; Petersen, J.; Neves, E. G. Soil Sci. Soc. Am. J., 2006, 70, pp 1719-1730 (DOI: 10.2136/sssaj2005.0383).

32. Steiner, C.; Teixeira, W. G.; Lehmann, J.; Zech, W. Microbial response to charcoal amendments of highly weathered soils and Amazonian dark earth in Central Amazonia: preliminary results. In: Glaser, B.; Woods, W. I. (Ed). Amazonian dark earths: explorations in space and time. Springer, New York, 2004, pp 195-212.

33. Novotny, E. H.; Azeredo, E. R.; Bonagamba, T. J.; Cunha, T. J. F.; Madari, B. E.; Benites, V. M.; Hayes, M. H. Environ. Sci. Technol., 2007, 41, pp 400-405 (DOI: 10.1021/es060941x).

34. Kern, D. C.; Costa, M. L.; Frazao, F. J. L. Geoquímica de sítio arqueológico com Terra Preta de Índio no centro da cidade de Quatipuru-PA. In: VI Simpósio de Geologia da Amazônia, Manaus, SBG, 1999, p 408.

35. Grimaldi, C.; Grimaldi, M.; Millet, A.; Bariac, T.; Boulegue, J. Hydrol. Process., 2004, 18, pp 93-106 (DOI: 10.1002/hyp.1314).

36. Brasil, E. C.; Cravo, M. S. Interpretação dos resultados de análises de solo. In: Cravo, M. S. et al. (Ed). Recomendações de adubação e calagem para o Estado do Pará. Belém, PA, Embrapa Amazônia Oriental, 2007, pp 43-48.

37. Viana, E. M.; Kiehl, J. C. Bragantia, 2010, 69, pp 975-982 (DOI: 10.1590/S0044-59672006000400001).

38. Fraser, J. A.; Clement, C. R. Bol. Mus. Para. Emílio Goeldi. Cienc. Hum., 2008, 3, pp 175-194 (DOI: 10.1590/S1981-81222008000200004).

39. Neves, L. S.; Ernani, P. R.; Simonete, M. A. Rev. Bras. Cienc. Solo, 2009, 33, pp 25-32 (DOI: 10.1590/S0100-06832009000100003).

40. Ernani, P. R.; Bayer, C.; Almeida, J. A.; Cassol, P. C. Rev. Bras. Cienc. Solo, 2007, 31, pp 393-402 (DOI: 10.1590/S0100-06832007000200022). 